\title{
EDITORIAL
}

https://doi.org/10.19053/22160159.v9.n19.2018.7919

\section{COTIDIANIDAD Y RETOS PARA EL PROFESORADO}

A propósito de los lugares y retos del maestro en la actualidad, surgen algunas cuestiones que pueden llegar a aportar al debate y reflexión que se orienta a las tensiones y distensiones en cuanto a la formación del profesorado, las funciones y condiciones de los maestros en ejercicio y al rol e incidencia social de la acción docente.

\section{La identidad}

La identidad profesional, que está relacionada en gran medida con la vocación, siempre ha sido muy importante, pero en un contexto educativo diverso, complejo y cambiante como el actual, es uno de los aspectos más relevantes, y debe ser abordada, trabajada y consolidada. La identidad profesional docente (IPD) integra rasgos de personalidad, motivaciones y actitudes (Martín-Gutiérrez, CondeJiménez \& Mayor-Ruíz, 2014). Además, su desarrollo se realiza a partir de la dimensión emocional, social-interpersonal, didáctica-pedagógica e institucional-administrativa (Buitrago-Bonilla \& Cárdenas-Soler, 2017). Por consiguiente, inciden en ella la promoción, los incentivos, el clima laboral, la configuración económica, política y social (Caballero \& Bolívar, 2015), el salario y la evaluación de desempeño (Roa, 2017).

La IPD es fundamental, debido a que permite que los maestros asuman su profesión como un verdadero estilo de vida (Sfard \& Prusak, 2005) y definan su propia manera de ser, actuar, asumir su trabajo y definir su estatus social (Sachs, 2005). Sumado a ello, cuando se aumenta la pasión por enseñar, se incrementa la satisfacción laboral y se disminuye tanto el estrés como el agotamiento, incidiendo por lo tanto en la calidad de las prácticas educativas y en los vínculos relacionales positivos (Carbonneau, Vallerand, Fernet \& Guay, 2008). 
En esta dirección, es muy importante que los programas de formación del profesorado incluyan en sus currículos el abordaje del desarrollo de competencias socioemocionales, superando la idea tradicional de plantearlas como competencias transversales (Buitrago, Ávila \& Cárdenas, 2017; Palomera, Fernández-Berrocal \& Bracket, 2008). Todo esto, debido a que la configuración de la identidad está orientada a la indagación y creación de sentidos y significados (Bedacarratx, 2012).

\section{La labor docente}

Preguntarse por la labor docente resulta complejo en los actuales escenarios sociales y educativos, mucho más si en algún momento se intenta establecer una postura concreta o un límite específico, ya que por un lado está la responsabilidad pedagógica, didáctica y educativa que por tradición tiene el docente; pero a esta se suma la transferencia de otro tipo de responsabilidades que la escuela ha ido asumiendo y que en otros momentos no le correspondían, y que quizá, por las actuales configuraciones sociales ha integrado como propias. Es así, como el conocimiento y las relaciones e interacciones generadas en la dinámica del aprendizaje han pasado a diversos planos, no siempre de protagonismo, para dar cabida a la atención de problemáticas psicosociales, económicas, familiares, administrativas y de diversas naturalezas.

En este sentido, Pulido y Gómez (2017) establecen que en las actuales condiciones es indispensable que el profesorado se adapte, contextualice y resignifique los conceptos a partir de los cuales estructura su acción docente. Y es que las aulas día a día se hacen más difíciles, las nuevas generaciones plantean nuevos desafíos y problemáticas que en muchas ocasiones el profesorado no logra dimensionar por completo, ya que las actitudes y comportamientos de los niños y adolescentes superan, por lo general, las perspectivas de los adultos, al incorporar la inestabilidad intrapersonal, la falta de seguridad, la baja autoestima, la adicción al móvil y a las redes sociales, las familias disfuncionales, las relaciones afectivas - y en muchas ocasiones sexuales—, los vínculos relacionales que no siempre son positivos y las múltiples presiones mediáticas a las cuales están expuestos. Por consiguiente, estas problemáticas se convierten en actores que se hacen presentes en la cotidianidad escolar para convivir y desafiar al profesorado. 
$\mathrm{Al}$ igual que la IPD, la formación de maestros es un proceso continuo que no tiene límite, que se consolida en la práctica educativa y que implica aprender y desaprender de acuerdo a las experiencias (Mariño, Pulido \& Morales, 2016). Sin embargo, en muchas ocasiones, pareciese que este aprendizaje no es suficiente, como tampoco la formación de los posgrados, ya que en muchas instituciones educativas los docentes y directivos docentes manifiestan de manera abierta que se sienten desbordados y piensan que los estudiantes están ganando el pulso y no saben qué hacer con ellos y sus conflictos, frente a lo cual se suele pensar que la respuesta está en el incremento del número de materias, contenidos y horas de dedicación académica, dejando en un segundo plano los vínculos interpersonales y el disfrute de cada una de las etapas del desarrollo.

A pesar de este panorama, aún hoy es escasa la presencia amplia, concreta y especializada del arte y la música en los currículos escolares, y mucho más de programas de desarrollo socioemocional. Al respecto, es sabido que tal y como ponen de manifiesto Herrera, Buitrago y Cepero (2017), se suelen encontrar diferencias en cuanto al desarrollo de habilidades emocionales de los niños de acuerdo con su género y contexto, lo que implica que la escuela debería dar una atención diferencial y no masificada y estandarizada a los alumnos.

\section{La tecnología}

El mundo ha experimentado en las últimas décadas un enorme incremento en cuanto al desarrollo tecnológico y su progresiva implementación. Estos recursos se han ido integrando a la dinámica escolar, o por lo menos eso es lo que se suele creer, sin embargo, aún son evidentes las brechas existentes entre distintas generaciones y la resistencia de un porcentaje considerable de docentes para dejarlas entrar a las aulas, vincularlas con sus prácticas educativas y articularlas con sus diseños didácticos, llegando incluso, en algunas ocasiones, a lo que Fuentes, Ortega y Lorenzo (2005) denominan tecnofobia.

Esta diversidad no solo se enfoca al uso de las TIC, sino que se hace evidente en la habilidad y conocimiento para realizar búsquedas y encontrar espacios con recursos y materiales que se puedan adaptar y utilizar en los desarrollos del aula. Y desde luego, se hace más complejo 
cuando se trata del diseño, creación y producción de este tipo de materiales y recursos tecnológicos. Lo cierto es que aún son reducidos, en muchos contextos escolares, el uso, el manejo y la integración de diversos recursos y posibilidades tecnológicas, como por ejemplo las aplicaciones.

\section{La lectura}

A pesar del énfasis que dan las pruebas estandarizadas a las habilidades lectoras, aún sigue siendo uno de los aspectos que más preocupa a los docentes y a las instituciones educativas, sin dejar de lado, desde luego, el ámbito escritural y de desarrollo del lenguaje. No obstante, en muchos sistemas educativos, en la práctica cotidiana, se sigue delegando esta responsabilidad a los docentes del área de lenguaje. Sin embargo, el desarrollo y disfrute de las habilidades de lectura, escritura y comunicación son indispensables para todo el proceso de desarrollo intrapersonal e interpersonal, formativo y de apropiación de conocimiento.

Para algunos autores, son tres los predictores que inciden en las habilidades lectoras: el procesamiento fonológico, el conocimiento de lo impreso y el lenguaje oral (Storch \& Whitehurst, 2002) y, es imprescindible que los niños aprendan a leer para que puedan llegar a leer para aprender (Senechal, LeFevre, Thomas, \& Daley, 1998). En este sentido, cuando los niños empiezan a leer, como es lógico, empiezan a apropiarse de nuevas palabras, conceptos, estructuras y narrativas que, sin lugar a dudas, mejoran y consolidan su desarrollo en el lenguaje (Justice, 2010). En cualquier caso, el interrogante fundamental es: ¿qué tanto disfrutan los niños y jóvenes sus lecturas?

En este sentido, es inevitable señalar que la motivación es fundamental y requiere de mayor atención, ya que el éxito de las habilidades lectoras y escriturales depende en gran medida de ella (Justice, 2010). Por lo tanto, el aprendizaje social y emocional es fundamental y debe ser implementado para aportar al desarrollo y estabilidad del estudiantado (Buitrago \& Herrera, 2013; Ekman, 2003; Herrera, Buitrago, Lorenzo, \& Badea, 2015), logrando con ello incidir de manera positiva en los procesos de apropiación y desarrollo de habilidades de lectura, escritura y lenguaje en los niños. Desde esta misma perspectiva, es fundamental 
poner de manifiesto la importancia pedagógica que, para el desarrollo de estas habilidades, tiene el cine, tal y como lo establecen Cañizales \& Pulido-Cortés, (2014).

$\mathrm{Al}$ respecto, es interesante enfatizar en el interés y disfrute que por la lectura manifiestan algunos niños y en especial adolescentes, quienes han llegado a devorar diferentes libros como los de Harry Potter de J. K. Rowling, la saga Crepúsculo de Stephenie Meyer, los libros de John Green, como Bajo la misma estrella, Buscando Alaska y Milveces hasta siempre, o iBuenos dias, princesa!, tengo un secreto: el diario de Meri de Blue Jeans, entre otros. Sin embargo, estos mismos estudiantes siguen considerando poco gratos en muchas ocasiones los libros, textos y documentos que son propuestos por los maestros en la escuela.

\section{Los procesos}

Si bien es cierto que en la educación contemporánea se busca alcanzar e implementar la flexibilidad en los currículos de los diferentes niveles, la actual configuración de muchos de los sistemas educativos, la sesgada visión de las políticas educativas y la baja inversión económica en investigación educativa, entre muchos otros factores, hacen que dicha flexibilidad tan solo sea, por lo general, una intensión difícil de alcanzar, una aspiración que moviliza algunas ideas, proyectos e intervenciones sin resultados significativos, o un ejercicio de apropiación teórica y conceptual. Por ejemplo, para el caso colombiano, el Ministerio de Educación (2018) señala que:

Un currículo flexible es aquel que mantiene los mismos objetivos generales para todos los estudiantes, pero da diferentes oportunidades de acceder a ellos: es decir, organiza su enseñanza desde la diversidad social, cultural y de estilos de aprendizaje de sus alumnos, tratando de dar a todos, la oportunidad de aprender (p. 1).

Lo cierto es que, en muchas ocasiones, los contenidos siguen siendo rígidos, las metodologías repetitivas, las perspectivas reducidas, el desarrollo de habilidades cuestionable y el disfrute del aprendizaje ocasional. Todo ello, porque la flexibilidad curricular implica una flexibilidad en múltiples niveles, como, por ejemplo, el normativo, el económico, el administrativo y el político, es decir, ante todo se requiere de una flexibilidad de pensamiento. 


\section{La estabilidad y la incidencia social}

La escuela es un lugar privilegiado, único y que posibilita una enorme diversidad de vínculos y relaciones, no obstante, el entramado que se configura en la actualidad ha integrado nuevos elementos o potenciado algunos que siempre estuvieron presentes. Estos factores se han hecho cada vez más visibles y han ganado protagonismo en la cotidianidad escolar, por lo tanto, han establecido otras formas de interacción y desde luego, nuevos desafíos para el contexto escolar, ya que ellos afectan tanto al alumnado como al profesorado y se relacionan de manera directa con lo que se siente y, por lo tanto, con la salud mental.

Es por ello que el estrés, la ansiedad y la depresión asisten de manera activa a la escuela, y se gatillan por la presión de los resultados, los diferentes tipos de acoso, el síndrome de desgaste profesional (burnout), los sentidos de vida difusos, los climas laborales tóxicos, los altos grados de corrupción, y la falta de coherencia de los gobiernos y en especial de quienes diseñan y orientan las políticas educativas. Desde esta perspectiva, es preocupante analizar el impacto social de la educación, y no se trata de señalar que es ella quien tiene la responsabilidad de la actual configuración, sino de entender que la educación al ser parte del sistema, es quizá la única alternativa posible para reconfigurar la estabilidad social.

\section{A manera de cierre}

Si bien es cierto que este es un momento en la historia en que se cuenta con un gran despliegue tecnológico, un amplio acceso a la información y al conocimiento científico; que se han consolidado prestigiosos programas de formación del profesorado en las universidades; que se aumenta la oferta de posgrados; y que los estados intentan establecer políticas apropiadas y contundentes para el desarrollo educativo de sus países - en muchas ocasiones de manera desacertada y sin propiciar las condiciones y destinar los recursos económicos necesarios-, es importante proponer algunos interrogantes relevantes: ¿qué tanto saben y profundizan los nuevos maestros en pedagogía? ¿Qué tan solventes son desde la perspectiva didáctica al culminar su formación profesional? ¿Al recibir su título cuentan con un adecuado desarrollo emocional? ¿Han realizado una configuración efectiva de su IPD? 
¿Cuenta con el conocimiento y habilidades tecnológicas suficientes? ¿Han desarrollado su creatividad, flexibilidad y adaptabilidad? ¿'Tienen un sólido compromiso intrapersonal, interpersonal y social?

Estos interrogantes adquieren relevancia porque el mundo es dinámico, en constante configuración y reconfiguración, y en las últimas décadas ha propiciado cambios. Aun así, los sistemas educativos se resisten a transformarse de manera eficaz e intentan implementar reformas parciales y en muchas ocasiones superfluas, que no dan respuesta a las reales necesidades personales, contextuales y sociales (Robinson, 2011). Por lo tanto, la eficacia y pertinencia educativa, la estabilidad social y cultural, así como la configuración de la autonomía, la libertad, la responsabilidad y la felicidad, están relacionados de manera directa con la formación del profesorado (Buitrago, 2016). Y son precisamente los maestros quienes, a pesar de los sistemas rígidos preexistentes, la normativa vigente — local, regional, nacional e internacional-, el escaso reconocimiento por parte de los gobiernos y las múltiples adversidades que se suscitan en la cotidianidad de la escuela, logran el privilegio y responsabilidad de tocar las almas de sus alumnos.

Para concluir, se presentan los artículos que integran el número 19 de la Revista Praxis \& Saber, los cuales profundizan en distintos aspectos que aportan a la reflexión, investigación y desarrollo del ámbito educativo. Esperamos que este nuevo número de Praxis \& Saber nutra, como siempre, el debate académico, la reflexión pedagógica y el quehacer investigativo.

Los autores David Andrés Rubio Gaviria y Rogger Mendoza Duarte, plantean una serie de argumentos en torno al lugar que tienen los maestros en la actualidad, enfatizando en tres posturas, la influencia de la psicología del desarrollo y el aprendizaje en la educación; la didáctica y su importancia en el aprendizaje de las disciplinas en la escuela; y el Campo Conceptual de la Pedagogía (CCP), en el artículo El aprendizajey el campo pedagógico: algunos conceptos fundamentales. El siguiente documento, El control de las prácticas escolares en la escuela Hondureña (1882-1899), de Andrés Eduardo García Lainez, presenta el análisis de la relación de la normativa en Honduras con las prácticas educativas que se realizaron durante este periodo, desde la perspectiva de las revistas educativas de esta época, abordando la acción del profesorado, la disciplina escolar, los métodos de enseñanza y la evaluación. 
En el artículo Estudio histórico-epistemológico y didáctico de la parábola, se presentan los resultados de una investigación realizada con estudiantes de la Universidad del Quindío, por parte de los autores Eliécer Aldana Bermúdez y Jorge Hernán López Mesa, en los que se analizan las maneras en que los estudiantes logran la comprensión y construcción del concepto de parábola, a partir de tres ámbitos, el históricoepistemológico, el didáctico y el cognitivo. La siguiente colaboración, de Luis Pérez Pinzón, Praxis didáctica inglesa y tecnología educativa alemana en el Estado Soberano de Santander, aborda el análisis del periodo de 1857-1886, focalizándose en el cambio del método lancasteriano por el pestalozziano, la incidencia de algunos pedagogos y la Misión Pedagógica Alemana, así como el uso de recursos de apoyo didáctico.

Por su parte, José Eduardo Cifuentes Garzón propone el manuscrito Movimiento en el aprendizaje de las rutinas de pensamiento en profesores, en el cual se analiza el pensamiento reflexivo de un equipo de profesores, así como su compromiso en las prácticas pedagógicas, todo ello, desde la perspectiva de la variación del aprendizaje respecto a las rutinas de pensamiento. Luego, se presenta el artículo Sentidos de formación, en las comunidades Achagua y Piapoco, de José Ignacio Bolaños Motta, Yamile Andrea Daza Monras y Kendy Viviana Rivera Barrios, en el que se explicitan los resultados de una investigación con dos comunidades indígenas colombianas del municipio de Puerto López, en el departamento del Meta, la cual se enfocó en conocer los sentidos de formación que se implementan en la escuela pública y los sentidos de adultez que se generan en los resguardos indígenas.

Para concluir el número 19 de Praxis \& Saber, se presenta, Teoría andragógica: aciertos y desaciertos en la formación docente en TIC, de Olga Sofía Morales Pacavita y Myriam Cecilia Leguizamón González, el cual, propone una interpretación respecto a los aciertos y desaciertos de la teoría andragógica en la formación de competencias tecnológicas del profesorado.

\author{
Rafael Enrique Buitrago Bonilla, Ph.D. \\ Universidad Pedagógica y Tecnológica de Colombia \\ Grupo de Investigación Cacaenta \\ Línea Emociones \& Educación
}




\section{Referencias}

Bedacarratx, V. (2012). Futuros maestros y la construcción de una identidad profesional: una mirada psicosocial a los procesos que se ponen en juego en los trayectos de formación en la práctica. Revista Electrónica de Investigación Educativa, 14(2), 133-149. Recuperado de: http://redie.uabc. $\mathrm{mx} /$ vol14no2/contenido-bedacarratx2012.html

Buitrago, R. E. (2016). La formación de maestros como alternativa para una educación de calidad. Praxis \& Saber, 7(15), 9-16. http://dx.doi. org/10.19053/22160159.v7.n15.2016.5720

Buitrago-Bonilla, R. E., \& Cárdenas-Soler, R. N. (2017). Emociones e Identidad Profesional Docente: Relaciones e Incidencia. Praxis \& Saber, 8(17), 225-247. https://doi.org/10.19053/22160159.v8.n17.2018.7208

Buitrago, R. E., \& Herrera, L. (2013). Matricular las Emociones en la Escuela, una Necesidad Educativa y Social. Praxis \& Saber, 8(4), 87-108.

Buitrago, R. E., Ávila, A. K., \& Cárdenas, R. N. (2017). El Sentido y el Significado Atribuido a las Emociones por el Profesorado en Formación de la Universidad Pedagógica y Tecnológica de Colombia. Contextos Educativos, 20, 77-93. http://doi.org/10.18172/con.2998

Caballero, K., \& Bolívar, A. (2015). El profesorado universitario como docente: hacia una identidad profesional que integre docencia e investigación. REDU, Revista de Docencia Universitaria, 13(1), 57-77.

Carbonneau, N., Vallerand, R. J., Fernet, C., \& Guay, F. (2008). The Role of Passion for Teaching in Intrapersonal and Interpersonal Outcomes. Journal of Educational Psychology, 100(4), 977-987. http://dx.doi. org/10.1037/a0012545

Cañizalez, N., \& Pulido, O. (2014). Infancia, una experiencia filosófica en el cine. Praxis \& Saber, 6(11), 245-262.

Ekman, P. (2003). La universalidad de las emociones. En D. Goleman (Ed.), Emociones Destructivas, cómo entenderlas y superarlas (pp. 71-93). Barcelona: Editorial Kairós.

Fuentes, J. A., Ortega J. A., \& Lorenzo, M. (2005). Tecnofobia como déficit formativo: Investigando la integración curricular de las TIC en centros públicos de ámbito rural y urbano. Educar, 36, 169-180.

Justice, L. M. (2010). La lectoescritura y su impacto en el desarrollo del niño: Comentarios sobre Tromblin y Sénéchal. En R. E. Tremblay, R. G. Barr, R. V. Peters, \& M. Boivin (Eds.), Enciclopedia sobre el Desarrollo de la Primera Infancia. Montreal (pp.1-4). Quebec: Centre of Excellence for Early Childhood Development.

Herrera, L., Buitrago, R. E., \& Cepero S. (2017). Emotional Intelligence in Colombian Primary School Children. Location and Gender. Universitas Psychologica, 16(3), 1-10. https://doi.org/10.11144/Javeriana.upsy16-3. eips 
Herrera, L., Buitrago, R. E., Lorenzo, O., \& Badea, M. (2015). Socio-Emotional Intelligence in Colombian Children of Primary Education. An analysis in rural and urban settings. Procedia - Social and Behavioral Sciences, 203, 4-10. http://dx.doi.org/10.1016/j.sbspro.2015.08.251

Mariño, L. A., Pulido, O., \& Morales, L. (2016). Actitud Filosófica, Infancia y Formación de Maestros. Praxis \& Saber, 15(7), 81-101. http://dx.doi. org/10.19053/22160159.v7.n15.2016.5724

Martín-Gutiérrez, A., Conde-Jiménez, J., \& Mayor-Ruíz, C. (2014). La identidad profesional docente del profesorado novel universitario. REDU, Revista de Docencia Universitaria, 12(4), 141-160.

MEN, (2018). Flexibilidad Curricular. Colombia. Recuperado de: https://www. mineducacion.gov.co/1621/article-82793.html

Palomera, R., Fernández-Berrocal, P., \& Bracket, M. (2008). La inteligencia emocional como una competencia básica en la formación inicial de los docentes: algunas evidencias. Revista electrónica de investigación psicoeducativa, 15(6) 437-454.

Pulido, O., \& Gómez, L. (2017). Del enseñar y el aprender. Praxis \& Saber, 8(18), 9-14. https://doi.org/10.19053/22160159.v8.n17.2018.7220

Roa, K. A. (2017). La evaluación docente bajo la óptica del desarrollo profesional: el caso chileno. Educación y Educadores, 20(1), 41-61. http:// dx.doi.org/10.5294/edu.2017.20.1.3

Robinson, K. (2011, 03, 16). Redes 87, El Sistema Educativo es Anacrónico [archivo de vídeo]. Recuperado de: https://www.youtube.com/ watch?v $=\mathrm{UlAs} 4 \mathrm{wPtBEU}$

Sachs, J. (2005). Teacher education and the development of professional identity: Learning to be a teacher. In P. Denicolo \& M. Kompf (Eds.), Connecting policy and practice: Challenges for teaching and learning in schools and universities (pp. 5-21). Oxford: Routledge.

Senechal, M., LeFevre, J., Thomas, E. M., \& Daley, K. E. (1998). Differential effects of home literacy experiences on the development of oral and written language. Reading Research Quarterly, 33, 96-116.

Sfard, A., \& Prusak, A. (2005). Telling identities: In search of an analytic tool for investigating learning as a culturally shaped activity. Educational Researcher, 34(4), 14-22.

Storch, S. A., Whitehurst, G. J. (2002). Oral language and code-related precursors to reading: Evidence from a longitudinal structural model. Developmental Psychology, 38(6), 934-947. 